\title{
One \\ SCIENTIFIC COSMOLOGY AND THE BIG BANG
}

Greek philosophers assumed that the world, the universe, the cosmos, or nature as a whole, existed in some form from eternity, that is, infinitely into the past, and that the basic stuff of the universe is uncreated, everlasting, self-sufficient, and indestructible. The official Christian view, by contrast, was and is that the world was created by God out of nothing (ex nihilo) at some point in the finite past. Naturalistic cosmologists through the centuries have sided with the Greek view and affirmed the everlasting self-existence and self-sufficiency of nature as a whole. Traditionally, each view was affirmed solely as a matter of dogma and blind faith, though Naturalists consistently intimated that science confirms their metaphysics. Up to the present century, however, no convincing evidence was available to resolve the dispute. Has this now changed?

In the twentieth century, cosmology tried to become a science, something more than mere speculation and dogma; and up to a point it succeeded. Twentieth-century cosmologists produced a plethora of astonishing discoveries about the universe as a whole. ' The most amazing is that the world of nature as we know it came into being at a definite point in the finite past. Our universe has not existed forever after all; it was created between 8 and 20 billion years ago. Do not conclude too hastily that God created it, or even that the origin of the world had a cause. Controversies about what, if anything, caused the Big Bang constitute the main subject matter of this book. We cannot address our central concern-What caused the Big Bang?-until we first survey evidence for the Big Bang and this theory's story of the evolution of the universe. Later, some significant challenges to Big Bang Cosmology will be confronted.

The astronomer Fred Hoyle, who did not favor the view, coined the apt pejorative phrase "Big Bang" for the theory that our universe began with the greatest thermonuclear explosion of all time in the finite but far distant past. In 1993, Sky and Telescope magazine ran a contest designed to find a better name for it, but after reading over 13,000 entries, some quite imaginative, the panel of prestigious judges decided that "Big Bang" is the best name after all. The winner of the contest was Fred Hoyle! As expressed in Sky and Telescope, "And the winner is...Nobody. Or Fred Hoyle, really, though he didn't submit 'the Big Bang' as an official entry last summer, having coined the term 44 years ago." Some imaginative losers were: "Hubble Bubble," "Planck Point," "Bertha D. Universe," "What Happens If I Press This Button?" and "You're Never Going to Get It All Back In There Again."'

As the theory developed in the twentieth century, differing estimates were given about how long ago the Big Bang happened. In the 1920s and 1930s, 
Edwin Hubble greatly overestimated the expansion rate of the universe; and this resulted in a significant miscalculation of the age of the universe-first at a billion years, and later at around two billion years. In 1952, George Gamow, an early popularizer of the view, published his The Creation of the Universe. Gamow calculated that the Big Bang occurred between 1.7 and 3.4 billion years ago. ${ }^{3}$ In 1958, Werner Heisenberg's Physics and Philosophy placed the origin of the universe at about four billion years ago. ${ }^{4}$ During the $1950 \mathrm{~s}$, available estimates of the age of the earth and its solar system showed them to be older than the universe, so something had to give!

Periodic improvements in methods for estimating cosmic distances and velocities bring about more accurate estimates of the time of the origin of the universe; but uncertainty lingers. The work of Allan Sandage and W. A. Baade in the late 1950s and early 1960s immensely expanded time/distance estimates in astronomy. In 1978, Robert Jastrow estimated the age of the universe to be around 20 billion years, give or take a few billion years one way or the other; 5 and Frank Tippler uses that figure in his 1994 book titled The Physics of Immortality. ${ }^{6}$ Today, most cosmologists accept 15 billion years as a workable compromise between the 8 to 20 billion year estimates generated by the inexact dating techniques and calculations available to today's astronomers.

Much more detailed observations and exact measurements by the Chandra X-ray Observatory launched in 1999, and the Hubble Space Telescope (deployed in 1990, repaired during 1993, and upgraded in 1997 and 1999), will yield a more precise time for the origin of the universe. At first, as many questions were raised as answered. Late in 1994, some data from Hubble observations suggested that the universe may be only 8 to 12 billion years old, in which case the age of some stars seems to be greater than that of the universe-perhaps twice as great! Still, most available data indicates a 10 to 18 billion-year-age. Astronomers scrambled to reconcile conflicting data, either by reintroducing an Einsteinian cosmological constant, by correcting the Hubble constant, by looking for ways to lower the estimated age of the stars, by developing more accurate measurements of celestial distances, or by greatly reducing estimates of the critical density or mass/energy in the universe. ${ }^{8}$ In early 1997 and thereafter, new and more accurate measurements of celestial distances were made public that harmonize the age of the universe with its stellar and galactic contents. ${ }^{9}$ With the availability of much more accurate measurements, by early 1999 something close to a fifteen billion-year-old cosmos seems very plausible..$^{10}$ I will use the convenient 15 billion-year figure for the age of the universe. Exact timing is not essential for identifying the ultimate cause of the Big Bang; what really matters is the well-founded scientific consensus that our world came into being somewhere between 8 and 20 billion years ago. In a later discussion of Big Accident Cosmology, the mass/density of the universe will be important for deciding whether the universe actually had a cause. 
According to Big Bang Cosmology, how did the universe evolve after its beginning, and what evidences support this sensational view of cosmic origins?

\section{The Evolution of the Universe}

Contemporary scientific cosmologists apply the concept of evolutionary development to the cosmos as a whole, not just to living things on earth. They are convinced that they can trace the broad outlines of cosmic evolution back to the beginning, around fifteen billion years ago, and that we can know more about the origin of the universe than about today's weather and earthquakes. The Standard Hot Big Bang Model prevailed between 1965 and 1980, when it was modified to take account of inflation and quantum effects. According to the Standard Model and a few widely publicized modifications, the universe as we know it developed as follows.

\section{A. The Initial Singularity}

Those of us who take pride in our common sense would be skeptical if told that in the distant past all of the material comprising the building in which we now live or work was once compacted into a ball no bigger than a basketball or a baseball. We would be astounded if further informed that at one time in the past, all of the matter/energy comprising the entire universe was compacted into a space no bigger than a baseball or a golf ball. Once, space itself was even smaller than that. We would be utterly incredulous to learn that in the beginning the totality of spacetime and all its contents were the size of a penny, a pinhead, or even a point. Yet, this is precisely what the Standard Model tells us, not as a matter of baseless dogma or mere speculation, but as grounded presumably in hard evidence. The historical development of Big Bang theory in the twentieth century will concern us only minimally. That story is well told by many other authors, ${ }^{11}$ but science cannot be divorced entirely from its history.

In the beginning, at $\mathrm{T}=0$, there was a singularity, according to the Standard Big Bang Model; and in this initial singularity, all the mass/energy of creation was compressed infinitely to zero size. The initial singularity had the volume of an Euclidean point, which has position but no magnitude at all; but, since no space existed in which it could assume a position, the initial singularity had neither position nor magnitude. In singularities, including the initial one and those that may exist in black holes and collapsed neutron stars, mass/energy is infinitely curved, infinitely hot, infinitely dense, and infinitely small. ${ }^{12}$ For the universe as a whole, at $\mathrm{T}=0$, the being of the world was as close to non-being as it could get! Perhaps, as some suggest, it just was non-being.

Between 1965 and 1970, two eminent British astrophysicists, Roger Penrose and Stephen Hawking, collaborated to prove that our universe began in a singularity, assuming that classical general relativity theory is correct. ${ }^{13}$ In his 
1988 best selling book, A Brief History of Time, Hawking reported that he had changed his mind; he wrote that he was "trying to convince other physicists that there was in fact no singularity at the beginning of the universe," that "It can disappear once quantum effects are taken into account." ${ }^{14}$ Like Hawking, many other contemporary cosmologists are greatly troubled by the initial singularity, as we will see in Chapter Four.

\section{B. Planck Time and Space}

Quantum Cosmologists trace the origin of the universe back to a tiny fraction of a second after the initiating explosion emerged from near nothingness, but they reject the initial singularity. Momentous developments occurred in the first milliseconds after creation, including the arrival of time, space, and all the laws of physics.

Planck Time is how long it takes a photon of light, which obviously travels at the speed of light, to traverse a unit of Planck Length or Space. In contemporary Quantum Cosmology, time and space are physically inseparable; and both have minimal quantum energy units, below which the very concepts of time and space have no meaning. These minimal units are called Planck Time and Planck Length or Space; at $10^{-43}\left(1 / 10^{43}\right)$ of a second, the size of the universe was $10^{-33}$ $\left(1 / 10^{33}\right)$ of a centimeter in diameter in volume. At those dimensions, Planck Density can also be calculated ( $10^{90}$ kilograms per cubic centimeter). The universe had to be at least that big and that old to exist at all. In Standard Big Bang Cosmology, by contrast, time and space can be condensed all the way down to a pointlike singularity; but Quantum Cosmologists think that physical time and space have no meaningful referent at less than Planck dimensions, ${ }^{15}$ and that it makes no sense to ask what happened before that in lesser quantities of spacetime. Yet, we will see, the question of "before that" is almost irresistible. Before that was a singularity, or the laws of quantum physics, or a vacuum fluctuation, or an antecedent universe, or Divine creation ex nihilo-or something.

Scientific cosmologists agree that all laws of physics break down or become totally inapplicable either at the initial singularity or at Planck Time. This includes the First Law of Thermodynamics, according to which energy is neither created nor destroyed. Laws of nature are employed in reasoning backward in time scientifically. If natural laws break down totally at an initial singularity or at the Planck Wall, nothing before that can be known scientifically because nothing remains to guide our extrapolations. As John A. Wheeler put it, "There never has been a law of physics that did not demand 'space' and 'time' for its statement.... With the collapse of space and time the framework falls down for everything one ever called a law of physics." ${ }^{16}$ The exact point of breakdown is a major controversy. If what Wheeler says is true, and if Planck spacetime is the point of ultimate collapse, extrapolating back to an initial 
singularity is impossible. Wheeler once did so, as we will see in discussing Oscillation Cosmology in Chapter Four; but this extrapolation assumes that the laws of physics continue to hold earlier than they can be known to apply, if Plank dimensions really are the ultimate limits of scientific knowledge.

\section{Inflation}

Several versions of Big Bang Cosmology were formulated early in the twentieth century, initially by Willem de Sitter in 1917 and Alexander Friedmann in 1922. ${ }^{17}$ Other interpretations were developed in the 1920s and 30s by George Lemaître, Arthur Eddington, and James Jeans. In the late 1940s George Gamow and his associates, Ralph Alper and Robert Herman, made major contributions. The Standard Model of the Big Bang based on relativity physics dominated astrophysics between 1965 and 1980. It affirms that the early universe emerged from a singularity and thereafter expanded at a fairly uniform rate.

No model published prior to 1980 made a place for inflation. What is inflation? Some scientific cosmologists now believe that when the universe was around $10^{-35}$ of a second old, it underwent a short but spectacular period of exponentially rapid expansion from Planck dimensions to roughly the size of a grapefruit. ${ }^{18}$ This lasted until $10^{-33}$ of a second after creation. Then the universe returned somehow (a great mystery) to the more leisurely and uniform pace of expansion predicted by the Standard Model. Alan H. Guth, who proposed the idea in $1980,{ }^{19}$ called this rapid expansion "inflation." Today, many cosmologists accept inflation, though not in the exact form originally proposed by Guth, not even Guth himself. Significant modifications of the inflationary scenario were proposed by Andreas Albrecht, Paul Steinhardt, and the Russian cosmologist Andrej Linde. ${ }^{20}$ Guth now acknowledges that inflation is more of a family of theories than a single theory of cosmic origins and recognizes more than fifty varieties. ${ }^{21}$ Some cosmologists are concerned that inflation may be so elusive that it could never be falsified, thus casting doubt upon its status as a scientific hypothesis. Although there is no direct empirical evidence for it, inflation is still widely accepted as a viable explanatory hypothesis because it is aesthetically appealing and provides plausible solutions to the horizon, magnetic monopole, and flatness problems. However, inflation theory may create the very problems to which it is supposedly the solution!

The horizon problem is the puzzle about why the observed universe is so homogenous and isotropic in structure and content, that is, why the universe is observed everywhere to be so remarkably uniform in temperature, background radiation, and large scale distributions of matter, despite the minor irregularities introduced by intermittent concentrations of matter in planets, stars, gaseous clouds, galaxies, and supergalaxies. Inflation suggests that the irregularities occurred when the universe was larger than the Standard Model could allow, and that the homogeneities were established when the universe was smaller than 
the Standard Model permits, thus enabling diverse parts of an initially tiny universe to influence one another causally and bring about the universe's observed uniformity. According to later versions of the theory, the universe did not begin homogeneously; but the chaotic perturbations and inhomogeneities that might have existed prior to the inflationary epoch were smoothed out by inflation itself. After inflation ended, additional fluke deviations or quantum fluctuations occurred; and these eventually carved out the stars and galaxies. ${ }^{22}$ Of course, inflation is not needed if the universe began homogeneously and isotropically, that is, if the uniformities were given as initial conditions.

The problem of magnetic monopoles is logically implied by Grand Unification Theories (GUTs), according to which four basic physical forces were unified as one force in the earliest trillionths of a second of creation. GUTs imply that massive numbers of large physical particles should exist with either a south or north pole, but not both. Such particles would be radically different from and incompatible with the existence of all physical particles identifiable today, which have both poles. According to Guth's first inflationary scenario, all the magnetic monopoles were exterminated during the inflationary era. Now he is convinced that this would not happen; and a new scenario is accepted because inflation's prediction of magnetic monopoles is "at odds with observation." In inflationary models, the production of magnetic monopoles is either avoided by delaying the genesis of material particles during a brief period of rapid supercooling that released the energy that caused inflation, ${ }^{23}$ or they are so dispersed by inflation itself that we just can't find them. None of this has been verified directly by observation; but supposedly the inflationary hypothesis is justified by its fruits, by its power to resolve otherwise intractable problems. Yet, it seems to create the very problems it solves, which otherwise don't exist! Inflation theory says that the universe should be rife with magnetic monopoles, but not one has ever been seen, and inflation theory tries to explain why nonexistent entities don't exist!

The flatness problem is the mystery over why space may be so little curved. It is closely related to the question of whether the universe contains so little mass/energy that it will continue to expand forever, or so much of it that gravity will eventually halt its expansion and bring about a contraction phase during which the universe slowly shrinks toward an ultimate Big Crunch. All the early versions of inflation predicted that the universe was blown out nearly flat at the beginning and that it is either just barely closed or perfectly balanced between open and closed. If these predictions cannot be sustained and confirmed, inflation is in deep trouble. As explained later, our universe looks very much like an open one, and inflation theorists are now scurrying (without great success) to develop models of inflation compatible with an open universe..$^{24}$

The issues of homogeneity, inhomogeneity, and the ultimate fate of the universe will be considered again later. Alan Guth believes that inflation is close to gaining universal acceptance, but it actually has many serious critics. Cosmol- 
ogists like Roger Penrose, ${ }^{25}$ A. Karel Velan, ${ }^{26}$ William L. Craig, ${ }^{27}$ and many other critics ${ }^{28}$ have serious doubts about inflation. Profound questions about it remain unanswered. Since scientists cannot repeat, verify, or falsify inflation, should it be taken seriously as a scientific hypothesis? Is it instead just metaphysics at its worst? Once inflation got underway, what brought it to a halt? How do we know that the universe was not just created by God or something else with initial conditions at the outset that insured general uniformity, the absence of magnetic monopoles, and whatever the curvature of space happens to be? Is inflation just a clever ruse for avoiding God? As Joseph Silk indicates, "Initial conditions are an alternative to inflation. Inflation is really a way of trying to erase arbitrary initial conditions. And it hasn't succeeded." ${ }^{29}$ By assuming arbitrarily that the conditions for which it wishes to account were not present as initial conditions for the universe, inflation theory creates all the problems that it tries to resolve.

Is the universe really as flat, as uncurved, as most theories of inflation imply? If so, does inflation really explain this flatness? If wrinkles and irregularities existed in early spacetime, why didn't inflation just blow them out into bigger wrinkles and irregularities? More seriously, how can inflation's usual presupposition that Omega (critical density) equals at least 1, that the universe is either closed or ultimately balanced between open and closed, be reconciled with empirical findings of only a tenth to a thirtieth or so of the mass/energy required to balance or to halt and reverse the expansion process? Peter Coles and George Ellis indicate that, though controversial, "An open [not a closed] universe might arise from inflation;" and they caution that "We do not in fact have any proof that inflation ever took place...." ${ }^{30}$ Considerations strongly favoring the openness of the universe will be presented later, especially in Chapter Three. At any rate, with or without inflation, the universe began about fifteen billion years ago, give or take a few billion here or there. With or without inflation, What caused the Big Bang? is a very good question.

\section{Evolution of Physical Forces, Particles, and the Laws of Nature}

During the remainder of the first minutes of creation, extremely important things happened that brought about the universe recognizable to us today, or at least to today's astrophysicists. During the first one to four minutes of time, the universe continued to expand and cool very rapidly; the four basic forces of physics separated from one another; the most elemental physical particles were formed; and the laws of nature came into being.

According to Grand Unification Theory (GUT), which aspires to be a theory of everything, the four basic forces of nature originally were only one force that divided into four at around $10^{-39}$ of a second. These physical forces are: 1 . gravity, the force that holds together large-scale objects like those perceptible by our senses; 2 . the strong nuclear force that holds protons and neu- 
trons together in atomic nuclei; it is immensely more powerful than gravity, but operates only at short distances within the nuclei of atoms; 3 . the electromagnetic force that holds electrons in orbit around atomic nuclei; and 4. the weak force that causes the slow decay of radioactive elements like uranium, ionium, radium, and radon. All of these forces are presumed to be mediated by messenger particles like gluons, gravitons, and $\mathrm{W}^{+}, \mathrm{W}^{-}$, and $\mathrm{Z}^{0}$. The last two of these elemental physical forces have now been combined into an "electroweak force;" but gravity is especially resistant to unification. At present, Grand Unification has not been confirmed; it is a hope, not a fact, an aspiration, not an accomplishment. When and if Grand Unification is achieved, this will be additional powerful evidence for the Big Bang.

Sub-atomic physical particles first emerged from the matterless energy of preceding phases of creation at around $10^{-6}$ of a second, but nothing as complex as atoms and chemical elements then existed. A dense unstable soup of photons plus sub-atomic matter and anti-matter particles evolved from pure energy. In particle accelerators, when particles are generated from energy, an equal number of matter and antimatter particles are always created. For this reason, astrophysicists believe that for a short time equal numbers of quarks and anti-quarks, neutrons and neutrinos, electrons and positrons, protons and antiprotons, and a vast plethora of particles and anti-particles were created in dense thermonuclear reactions in the very early universe. None of these particles and antiparticles endured for very long. When they collided, they annihilated one another, released more energy, and generated additional radiation and sub-atomic particles. By the end of the first thirty minutes, by some process mysterious even to astrophysicists, the symmetry of matter and antimatter in the universe was broken. Somehow matter came to predominate over antimatter; and thus we live in a world of matter, not antimatter.

Natural laws describe general features of structures and processes in the world of public, perceptible spacetime. Laws of nature that apply to the four basic physical forces and to diverse sub-atomic, atomic, chemical, biological, psychological, and social entities came into being along with the realities whose patterns they depict. Through the centuries, the precise kind of reality possessed by natural laws has been much debated. A once popular view, now outmoded, affirmed that natural laws are powers or forces that compel things to be what they are and do what they do. The dominant view today is that laws of nature are merely statistical patterns that describe but do not forcefully compel or restrain natural structures and processes. ${ }^{31}$ Natural laws have no causal efficacy in themselves; they do not cause things to exist; they neither force things to do what they do nor to be what they are; and they do not impose external restraints. At best, natural laws are only formal causes, not efficient causes. They do not even exist prior to the things that they "regulate" or "govern." They tell us nothing about the behavior of a single entity like a single atom or molecule; they merely summarize the average behavior of actual things in large groups. They 
do not describe the activities of individuals with absolute precision. Individuals come first; and their existence, natures, activities, and habits gradually engender recognizable statistical patterns. Knowledge of these general configurations by intelligent beings comes much later. Laws of nature merely describe how things behave on the average, as we discern them. Until entities exists in quantity, their configurations and the laws that portray their functional patterns do not exist, except perhaps as possibilities or exemplars in the mind of God. The real significance of natural laws is not that they are efficient causes but rather that they permit us to reason from the observed to the unobserved. Our abilities to predict and control future events and to understand past events depend upon this reasoning. Natural laws are only formal and probabilistic, not efficient and exact, causes of groups of physical events. They do not pre-exist these events to impose anything upon them.

Natural laws operate within given physical conditions. Big Bang Cosmology prompts astrophysicists to inquire about the initial conditions of the universe. What kind of habituated or habit-forming stuff was given originally for the laws of nature to describe? Attempts to dispense with initial conditions by converting or reducing them to natural laws seem doomed to failure. The quantity of mass/energy in the universe, extremely low entropy or disorder, no more than four basic physical forces, their relative strengths, permissible kinds of physical particles, the directionality of time, and other conditions resulting in regular or lawful patterns of events were simply given at the beginning of creation. These primordial conditions spawned numerical constants of nature like the relatively unchanging numbers associated with the four basic forces, Planck's constant, charges for electrons and protons, definite masses for different kinds of physical particles, the rate of Hubble expansion, and the speed of light. Physical constants are extremely useful to us in predicting past and future courses of events. Natural laws merely describe the formal patterns of processes and realities in nature; but in themselves they are devoid of energy or power.

\section{E. From a Universe of Radiation to a Gaseous Universe}

The nuclei of hydrogen and helium atoms were formed when the universe was about one second old; but for the first 700,000 years, the cosmos, composed of turbulent radiant energy, was almost without form and void. Stable atoms could not form during the first part of this radiation era because electrons were constantly being knocked out of place from their orbits around protons. The "primordial fireball," as it is often called, was at first completely dark and fireless for many hundreds of thousands of years. After around 300,000 years, atoms began to form as the universe further expanded and cooled. Until then, darkness was upon the face of the deep; but as atoms formed, more space was created, and photons were set free to illuminate the universe for the first time; and suddenly, after 300,000 years or so of darkness, there was light. The all- 
pervasive microwave background that we detect today reflects these processes and originated during this era of cosmic evolution. Finally, after 700,000 to a million years, the universe became a dense gaseous cloud consisting of about 75 percent hydrogen and 25 percent helium, with traces of deuterium and lithium. This gaseous plasma continued to expand and cool; but it contained no heavy elements. No quasars, galaxies, stars, or planets graced the skies.

\section{F. Creation of Quasars, Galaxies, Stars, Solar Systems, Heavier Elements}

When the gaseous universe was a billion or so years of age, two powerful forces and an initial irregularity or fluke fluctuations finally separated and produced massive objects in the heavens. The two forces were (1) the kinetic expansive energy of the original cosmic explosion that continued to dissipate the hydrogen and helium gases and (2) the opposing attractive force of gravity that slowly assembled huge masses of hydrogen and helium gasses to form the stuff of millions of quasars and supergalaxies, plus billions of galaxies and their innumerable stars.

Either irregularities were built into the universe as initial conditions, or quantum flukes explain why, despite gravity, the gaseous universe did not expand indefinitely as a homogeneous gaseous plasma. Most astrophysicists today think that quantum physics accounts for these flukes. Just where quantum physics begins to apply to the early universe is highly problematic. Some think it doesn't apply at all because they interpret quantum physics only phenomenologically or epistemologically as informing us only about how quantum conditions appear to us, not about how they really are or were.

As better explained in Chapter Six, quantum physics is interpreted in this book as applying realistically and ontologically to the actual structure of the physical world. Some ontological realists think that quantum physics applies at or very near the beginning of creation, perhaps at or immediately following the very earliest Planck or inflationary moments; others think that it begins to apply only when quantum fields of energy or tiny individuated quantum objects like electrons and photons made their first appearance. In any event, minuscule quantum fluctuations occurred at some point in the very early universe, and these significantly affected the density and distribution of existing mass/energy in the later universe. Eventually these perturbations had massive cumulative effects. Persisting and spreading for a billion years or more, the initially small effects of very early sub-microscopic vacillations caused huge clouds of gas to separate. Then gravity pulled their ingredients together to form quasars, galaxies, and stars.

Although only .002 percent of all existing hydrogen is heavy hydrogen or deuterium, that tiny amount is indispensable for igniting all stellar furnaces. Gravity alone is too weak to condense and heat the hydrogen in evolving stars to the temperature required to ignite the process of nuclear fusion that drives all 
stars and suns; but gravity can and did produce the lower densities and temperatures required to initiate deuterium fusion. Deuterium fusion then inaugurated the fusion of hydrogen into helium, and flaming galaxies, quasars, stars, and suns graced the skies many hundreds of thousand of years after the initiation of the Big Bang.

\section{G. Formation of Our Sun, Its Planets, and Life on Earth}

Aristotle and Ptolemy believed mistakenly that the heavens were absolutely perfect in their changelessness. They and their ancient and medieval disciples could see only the deceptive stability of stars in our Milky Way and a few "wandering stars," the planets. Unknown to the ancients, many other galaxies of stars exist, and so did earlier generations of stars. Eventually, some of these stars, the giant supernovas, exploded and filled the heavens with stardust.

Five billion or so years ago, ten billion years after the onset of the primordial Big Bang explosion, gravity assembled enough stellar debris to form our sun and its planets, including our earth. Our sun presently has enough nuclear fuel to burn for another five billion years, so it is a middle-aged star. The earth existed for about one to one and a half billion years before the most primitive forms of life appeared. Don't ask how! No one really knows! All the oxygen, carbon, nitrogen, iron, and other heavy elements that constitute our bodies and those of all living things were forged earlier in stellar furnaces. No life could have formed anywhere during the first generation of stars composed almost entirely of hydrogen and helium. Supernovas first had to manufacture the heavier elements, then explode to scatter their stuff of life into the cosmos. The process is cumulative, so later and later supernovas consist of more and more metals and other heavier elements. Our sun, all its planets, and we ourselves were fabricated by gravity out of the rubble of numerous supernova explosions. As William Fowler said, "Each one of us and all of us are truly and literally a little bit of stardust." ${ }^{32}$ This may explain our fascination with and Immanuel Kant's being filled with awe by "the starry heavens above." We are all stuff of their stuff.

Assuming a 15 billion-year-old universe, about three and a half to four billion years ago, eleven and a half to twelve billion years after the Big Bang was initiated, microscopically small forms of life first appeared on earth. Much later, after more than 2,500,000,000 years of evolution, our earliest uprightwalking hominid ancestors, members of the species Australopithecus anamensis, first emerged in Africa around 400 to 300 million years ago, more than 14.5 billion years after the Big Bang. They and their descendants like Australopithecus afarensis and Homo erectus flourished for well over a million years. Homo erectus migrated from Africa into Asia and Europe. Exactly when the earliest members of our own hominid species, Homo sapiens, descended from them and first appeared is a matter of great controversy; but it happened somewhere 
between 500,000 and 100,000 years ago, most probably around 200,000 years ago. We are newcomers in creation.

\section{Evidences for the Big Bang}

What evidences support the Big Bang account of the origin and development of our cosmos? Why do scientific cosmologists believe that our universe began in a cataclysmic thermonuclear explosion? Evidence accumulated slowly for most of the twentieth century. Today it is so overwhelming that almost all contemporary astronomers, astrophysicists, and scientists are convinced that our world origi-nated in an astronomical explosion at some point in the finite past. Its present structure evolved from a primordial fireball. Only a very few cosmologists like Eric Lerner are unconvinced. His serious challenge to Big Bang Cosmology will be discussed in Chapter Three.

Explosions familiar to us take place within a pre-existing spacetime system, and their debris scatters into that system; but the initial Big Bang was very different from familiar bomb and dynamite blasts. The Big Bang created spacetime itself. Space is not sheer nothingness, as common sense tends to assume; it has its own physical properties. It is a primordial physical medium that has its own energy density and texture. It is grainy, granular, or foamy; and it is elastic, temporally expanding, but capable of being shrunk, bent, knotted, and warped. All physical things, including explosions, are manifestations of it. The following converging lines of evidence imply that the basic spatiotemporally extended stuff of our universe originated in a thermonuclear explosion around fifteen billion years ago.

\section{A. Receding Galaxies and the Redshift}

Only since the early 1920 s have we known with assurance of the existence of other galaxies, profuse with stars. Before powerful telescopes were built early in this century, astronomers could not see any individual stars beyond our own galaxy. They could see a few nebulae, but they could not confirm that they are composed of stars or that they exist beyond our Milky Way. With the naked eye, we can only see individual stars in our own galaxy, the Milky Way with its hundred billion suns. We can also see a few dim nebulae, like the nearby Andromeda Nebula, whose composition was unknown before the 1920s.

With his feeble telescope, Galileo could see the moons orbiting Jupiter. He was the first to see that the Milky Way is composed of individual stars, but he could not see any single stars within the hundred and twenty five billion or more galaxies that lie beyond our own Milky Way. By the middle of the eighteenth century, telescopes were powerful enough to see many nebulae; and in 1784, Charles Messier published a list of 103 bright clusters and nebulae, some of which turned out to be extragalactic. ${ }^{33}$ Thomas Wright conjectured as early as 
1750 that the spiral nebulae are clusters of stars. In 1850, Baron Alexander von Humboldt called them "island universes." ${ }^{34}$ Without empirical verification, most astronomers accepted this view from Messier's day to late in the nineteenth century, when a few influential astronomers shifted to the position that they are only clouds of gas located within the Milky Way. ${ }^{35}$

Edwin Hubble first confirmed the island universes hypothesis. Beginning early in the twentieth century, astronomers employed much more powerful telescopes to probe the distant universe. In 1924, Edwin Hubble, using the 100inch telescope at Mount Wilson Observatory in California, confirmed that the nebulae contain individual stars and that giant galaxies of stars exist beyond the Milky Way, our local galaxy. Hubble developed methods for measuring cosmic distances based upon the luminosity of "standard candles" in the sky, but we now know that his original computations greatly underestimated these distances and the corresponding age of the universe. He proved, nevertheless, that the universe is immensely larger and richer in contents than most people had ever dreamed it to be.

Edwin Hubble and Milton Humason, his associate, made and reported numerous observations that confirm another startling truth about the galaxies, the redshift; but they were not the first to see and discuss it. V. M. Slipher at the Lowell Observatory noticed the redshift in 1912 while observing and charting a small number of spiral nebulae, but he did not realize that they are clusters of stars and stardust, and that their redshift evidences an expanding universe.

What is the redshift, and what is its significance for the origin of our universe? In the middle of the nineteenth century, Christian Doppler discovered some important truths about light emitted by moving objects. If light coming from an object moving toward an observer is filtered through a spectroscope, its wavelength shortens and shifts toward the blue end of the spectrum. If the object is moving away from the observer, its wavelength lengthens and shifts toward the red end of the spectrum. Radar guns used today by traffic police make use of this "Doppler effect." Light waves coming from distant objects moving rapidly away from us are stretched toward the red end of the spectrum; and the further away and faster these objects are, the redder the shift. The degree of this shift is proportional to their speed and distance from the observer.

Hubble and Humason examined numerous galaxies and found in most cases that they manifest the "Doppler effect," the redshift; and they inferred that most observable galaxies in the universe are moving away from us. This was, as they recognized, the first hard empirical evidence that the universe expands as time marches on. The redshift of the galaxies does not result from their moving through space like projectiles, but from the expansion of space itself.

Galactic redshift is extremely important evidence for Big Bang Cosmology. If the spatiotemporal universe expands as it moves into the future, then earlier and earlier in the past it must have been more and more compacted or concentrated. The calculable rate of cosmic expansion, expressed in Hubble's 
1929 law of uniform expansion, implies a relatively uniform rate of contraction, applied retroactively. Calculations reveal that at some point in the finite past, the universe was compacted to zero, an initial singularity; but this implication did not become clear for some time. Much later in the century, quantum physicists discerned that all laws of nature break down or become inapplicable at less than Planck time. If so, compaction below Planck dimensions to an even earlier initial singularity cannot be inferred after all; but that story will be told later.

Hubble found that although most galaxies manifest the redshift, a few do not. Our nearest neighbors, the Andromeda galaxy, the Magellanic Clouds, and twenty five or so other small galaxies nearby, do not exhibit the redshift because they are sufficiently close to be gravitationally bound to our Milky Way. In 1994, astronomers discovered a large, nearby, and previously unknown galaxy hidden behind our Milky Way; and there may be others. ${ }^{36}$ The Andromeda galaxy, with a blueshift, is actually heading towards us and will collide with our Milky Way in about five billion years. This should not cause alarm, however; neither we nor our descendants will be around to see it; and it may only pass through the Milky Way with few collisions between vastly separated stars.

\section{B. Hubble's Law of Uniform Expansion}

In 1924 Edwin Hubble established that most galaxies are moving away from us; and by 1929 he realized that they do so in a lawlike manner. They move away from us and from one another at a uniform and calculable rate, like raisins in a rising loaf of bread, or dots on the surface of an inflating balloon. According to Hubble's law of uniform expansion, in a homogeneous universe, galaxies move away from us and from one another at speeds proportional to their distance.

Improved contemporary estimates of cosmic distances and velocities differ significantly from the results of Hubble's initial computations. We now know that the galaxies will double their distances from one another and from us in less than ten billion years. Before late 1997 or early 1998 astronomers assumed that the rate of cosmic expansion is being slowed by gravity, so it was much more rapid in the distant past when cosmic distances were smaller, and it will be much slower in the far distant future when the size of the universe is significantly greater. It now appears, as explained in Chapter Three, that the rate is accelerating, not slowing. This rate of expansion, known as the "Hubble constant," remains nearly the same during the lifetime of any human astronomer; but from a cosmic perspective, the pace of intergalactic distancing is anything but constant. Very recent work pins the Hubble constant down to between 60 and 75 kilometers per second for each megaparsec (3.26 million light years) of distance. ${ }^{37}$

The reverse of expansion is contraction. Hubble's law is significant for Big Bang Cosmology because it implies that in the finite but far distant past, all the mass/energy in the universe, including all matter in all the galaxies, was 
compressed together and belonged to one exploding primordial fireball. Even earlier, it was compacted either to zero, an initial singularity, or to Planck or other finite dimensions. If the laws of nature collapse at or beyond the Planck Wall, nothing smaller or earlier could exist as a part of our universe.

\section{The First and Second Laws of Thermodynamics}

The law of increasing entropy affirms that order and energy are constantly being lost or dissipated in closed systems that are not drawing and renewing them from elsewhere. Loss of order and energy is irreversible and time-asymmetrical. Applied to the whole universe as a closed system, this law indicates that disorder increases universally, and perhaps that overall energy concentrations decrease, as the cosmos expands and cools over time. It also implies that the energy and order of the universe were more and more concentrated and organized earlier and earlier in time. The universe began in a state of low entropy, and if it were infinitely old it now would be in a state of maximum entropy. However, since it is not infinitely disordered, it cannot be infinitely old. At some point in the finite past, about fifteen billion years ago, the universe was totally concentrated into a singularity or to Planck or other minute dimensions.

The First Law of Thermodynamics affirms that energy is conserved, that it can be neither created nor destroyed, that it can only be transformed into other types. This well verified law of nature implies nothing metaphysical about the ultimate origin of the mass/energy of the universe. It does not imply that mass/ energy existed everlastingly and necessarily throughout an infinite past, though it is occasionally given this metaphysical interpretation. ${ }^{38}$ Standard Big Bang Cosmology affirms that all the mass/energy of creation came into being with its laws about fifteen billion years ago. As a law of science, the First Law of Thermodynamics says simply that once created, energy is conserved and that we know of no physical way that we can destroy it. Superficially, the First Law of Thermodynamics, which says that mass/energy cannot be created, appears to conflict with Big Bang Cosmology, which says that all the mass/energy of the universe was created around fifteen billion years ago. However, the empirical or scientific claim that "The amount of energy in the universe is constant" should not be confused with the metaphysical claim that "The amount of energy in the universe is necessary, uncreated, self-sufficient, and everlasting." If our universe was actually preceded by an earlier universe that gravitationally collapsed to a singularity, all previous conservation laws would have collapsed and terminated as it crunched to spatiotemporal nothingness. As Charles Misner, Kip Thorne, and John A. Wheeler say,

Of all principles of physics, the laws of conservation of charge, lepton number, baryon number, mass, and angular momentum are among the 
most firmly established. Yet, with gravitational collapse the content of these conservation laws also collapses. The established is disestablished. ${ }^{39}$

Conservation laws are really not incompatible with Standard Big Bang Cosmology because the conservation of mass/energy, like all other laws of nature, begins with and does not antedate the Big Bang.

The Second Law of Thermodynamics applies fully only to closed systems that do not draw energy and order from other systems. The growing bodies of living things, including living human beings, concentrate order and energy; but this does not violate the Second Law because all living things eat, drink, respire, photosynthesize (if plants), and draw their sustenance from outside themselves. They are not completely closed systems, so the Second Law does not apply fully to them. Considered apart from the energy that they draw from elsewhere, the law does apply. Animal bodies constantly lose and expend ordered energy, which is why they require renewal meal after meal, breath after breath. If not fed, they starve. Plants also lose ordered energy and renew it through photosynthesis and by absorbing nutrients through their foliage and roots.

The concept of "entropy" may involve subtle ambiguities. Roger Penrose notes that "Entropy is a concept that may be banded about in a totally cavalier fashion!" ${ }^{40} \mathrm{He}$ suggests that there may be something subjective about the "order" involved in low entropy, that "Various observers' aesthetic judgments might well get involved in what they deem to be 'order', rather than disorder."

Increasing entropy is decreasing order, so to understand it we must advance and comprehend a concept of order. Contemporary discussions of entropy give many different accounts of what counts as order. Astrophysicists identify order with the undifferentiated homogeneity of mass/energy in the earliest universe, with matter that has not yet been converted into pure or unavailable energy, with an intense concentration of energy, with the relatively low ratio between the number of photons and the number of other particles in the universe, with the ability to recover information from antecedent states of affairs, with energy states not pervaded by destructive ripples, with complex environments that can support intelligent forms of life, and perhaps with all of these. But can all of the above be consistently combined? Measured by the standard of being life-supporting, the earliest universe was chaos, high entropy, great disorder, "without form and void." Yet, it was low in entropy when order is identified with the original concentrated and homogeneous soup of radiant energy or sub-atomic particles that ultimately produced an available-energyenvironment supportive of and usable by intelligent life. Whether the very early universe is characterized as ordered or chaotic may be a matter of wording and emphasis. George Gamow opted for chaos or high entropy when he wrote that "In the distant past our universe was considerably less differentiated and complex than it is now and the state of matter at that time could be accurately described by the classical concept of 'primordial chaos." ${ }^{42}$ This is really not 
incompatible with Roger Penrose's emphasis, discussed later, on low entropy as an initial condition of the universe because Penrose employs different concepts of order and entropy.

Entropy is often conceived as a decrease in the concentration of both energy and order, but these are two very different things. In the expansion phase of our universe, they normally hang together, but not necessarily, not always, not in the contraction phase if one is to occur, and not in contemporary regions of gravitational collapse. Neutron stars are so massive and dense that gravity has fused their electrons and protons into neutrons. In black holes or collapsing neutron stars or universes, energy concentrates but disorder increases. Neither black holes nor collapsing universes violate the First Law of Thermodynamics, for they are not losing energy absolutely. Spacetime and energy are continuously constricted and concentrated in them while life-supportive order, available information, usable energy, and recoverable information deteriorate, and destructive ripples increase, so most astrophysicists now believe; but the issue is contested.

With respect to energy, black holes as well as collapsing stars and universes actually do violate the Second Law of Thermodynamics, the law of increasing entropy, for energy concentrates in them; but with respect to life supporting, ripple-free usable energy and available information, they do not violate this law, for these elements of order decrease within them. Concerning order, but not energy, Roger Penrose affirms that "The second law will hold sway just as much inside a black hole as it does elsewhere." ${ }^{43}$ By this, Penrose means that human existence in black holes and collapsing universes would be utterly impossible because the kind of order that could support us would be lost and lacking.

Penrose thinks that no living space traveler could survive being sucked into a black hole; the violence and disorder would be unendurable. ${ }^{44}$ Reflecting on recollapsing universes, Stephen W. Hawking agrees and confesses,

At first, I believed that disorder would decrease when the universe recollapsed. This was because I thought that the universe had to return to a smooth and ordered state when it became small again. This would mean that the contracting phase would be like the time reverse of the expanding phase. People in the contracting phase would live their lives backwards: they would die before they were born and get younger as the universe contracted..$^{45}$

After reading a brilliant article by Don Page titled "Will Entropy Decrease if the Universe Recollapses?"46 Hawking decided that he had made a mistake. He came to believe that "disorder would in fact continue to increase during the contraction" and that "Conditions in the contracting phase would not be suitable for the existence of intelligent beings...." ${ }^{\prime 47}$ On this view, in black holes and 
collapsing stars and universes, energy concentrates; but life-supporting order, available information, and ripple-free usable energy do not. In his work on radiating black holes, Hawking contends that the wave functions of all objects sucked into black holes, and thus all information about them, would be lost irretrievably. Entropy in this sense would consist in the loss of wave function probabilities and information about them. Yet, some astrophysicists are not fully convinced and argue that entropy would decrease (meaning presumably that both energy and order increase) in a collapsing universe ${ }^{48}$ and that information initially lost into a black hole might be recovered from the radiation it slowly emits. ${ }^{49}$ This issue will arise again in later discussions.

\section{Inferences from Einstein's Theory of Relativity}

In 1905, Albert Einstein developed his special theory of relativity. It denied the reality of absolute Newtonian time and space, which were always and everywhere the same. It affirmed that space and time are inextricably united; no timeless space or spaceless time can exist. Their apparent constancy depends on the speed at which observers and their immediately surrounding spacetime frames are traveling. The speed of light is Einstein's only constant; observers always find it to be the same no matter where they are or how fast they are moving; but this implies the relativity of Newton's absolute constants, space and time.

In 1915 Einstein developed, and in 1917 he published, his general theory of relativity. It was a new theory of gravity and curved non-Newtonian space. Einstein regarded them as identical. General relativity affirms that space itself, and the path of light waves and other particles in terms of which we measure space, are curved or distorted in the presence of omnipresent gravitational fields. Light travels in curved paths in such fields. Space is more than a homogeneous empty form; it is something real in itself that can be concentrated, stretched, shrunk, bent, and straightened. The curvature of space just consists of gravitational fields that vary in intensity with variations in mass.

To Einstein's surprise and dismay, when combined with Hubble's observations of the redshift, his relativity field equations indicated that the universe is actually finite but expanding with unbounded potential, and that it had a beginning in time. When he realized this, Einstein was horrified! He mistakenly assumed that a finite universe must have an absolute center, and this was prohibited by his special theory of relativity. The Copernican Principle affirms that there are no privileged positions and observers in a relativity universe.

Einstein was both a theoretical physicist and a philosophical metaphysician. When he first discovered his general theory of relativity, he believed with Spinoza in a universe that is static, uniform, infinite, and eternal. Robert Jastrow tells us that, "When Einstein came to New York in 1921 a rabbi sent him a 
telegram asking, 'Do you believe in God?' and Einstein replied, 'I believe in Spinoza's God, who reveals himself in the orderly harmony of what exists." "50

Most Theists have a suspicion that Spinoza's God is no God at all. Spinoza's God is Nature, and Nature is his God. Spinoza did not deify nature; he naturalized deity. To believe in Spinoza's God is to affirm that the universe is penetrable to reason, that determinism reigns inexorably, that "God does not play dice" (as Einstein put it), and that the world is spatially infinite, eternal, necessary, unchanging, and static. Initially, Einstein accepted this metaphysics, believing that reality is timeless and changeless, and that human distinctions between past, present, and future are illusions. Einstein once wrote that "Space and time are not conditions in which we live, but modes in which we think." 1 Jastrow asks: "Why did Einstein object to the idea of a beginning?" and he answers:

I think it is plausible that he did not believe in God the Creator. A beginning presupposes an agent that set in motion the events, which we call the explosion of the universe. That was anathema to Einstein. He believed in Spinoza's God, who created order and harmony in the universe and is revealed in equations like Einstein's relativity equation, but he did not believe in a personal God or God the Creator. ${ }^{52}$

Given his metaphysical inclinations, it is small wonder that Einstein reacted with powerful negative emotions when Willem de Sitter, a Dutch astronomer, showed him in correspondence during 1917 that his relativity equations have non-static solutions which imply an expanding universe that originated in the finite past. According to Jastrow, Einstein wrote back, "This circumstance irritates me" and "To admit such possibilities seems senseless." ${ }^{53}$ Notice, says Jastrow, the emotionally loaded language! When Alexander Friedman, a Russian mathematician, proved that Einstein's rejection of an expanding universe was based on a mistake in calculation, Einstein first ignored him, then attempted to prove him wrong, and finally published a confession of his error. ${ }^{54} \mathrm{After}$ Edwin Hubble decisively confirmed the redshift of the galaxies, Einstein refused to accept an expanding universe until he traveled from Germany to California in 1931 to see for himself. Then theory yielded to facts.

Einstein finally realized that his preferred static but finite universe would collapse under the weight of its own gravity; so he postulated a "cosmological term" as an equal repulsive force that would prevent this collapse. Later, he confessed that this was the greatest mistake of his career. It prevented him from discovering the Big Bang.

What is the nature of this repulsive force that might so nicely counterbalance gravitational attraction? Einstein had no answer. Big Bang Cosmologists know that a repulsive force does operate in the universe-the kinetic energy left over from the primordial explosion; but whether there is an additional repulsive 
force, a Cosmological Constant, is still being debated. For billions of years, the repulsive force of energy released by the Big Bang has exceeded the cohesive force of gravity. It may or may not do so always. The ultimate destiny of the universe hangs in the balance. Stay tuned for breaking developments!

\section{E. Nucleosynthesis of Hydrogen, Helium, Deuterium, and Heavier Elements}

In contemporary cosmology, astronomy merges with sub-atomic particle physics. Astrophysicists now believe that the smallest things in the universe can tell us something significant about the universe as a whole, and vice versa. Research with particle accelerators discloses both the laws of physics and the conditions under which stable atomic elements were created out of sub-atomic particle soup in the very early universe. Alchemists' dreams of converting one element into another come true in nuclear physics, but only under extremely exotic conditions. Particle physicists recognize that some states and processes predicted by their theories were realized only under the extreme conditions of density and temperature of the Big Bang, and in the stars as nuclear furnaces.

Conditions were right in the very early universe for the nucleosynthesis of hydrogen into helium, but not for producing the heavier elements. Big Bang Cosmology predicts that the universe consists of about 75 percent hydrogen and 24 percent helium. Spectroscopic examination of countless stars and galaxies confirms this prediction, which is powerful evidence for Big Bang Cosmology.

The remaining heavier elements, quite abundant in our local terrestrial environment, constitute only about one percent of the stuff of the universe. In 1950 , George Gamow argued that the heavy elements were created very shortly after the initial explosion as the universe expanded and cooled. However, research with nuclear reactions and "atom smashers" shows that conditions in the earliest universe could generate only the prevailing hydrogen and helium abundances. Also, if all of the radioactively unstable elements had originated in the Big Bang, they would have completely decayed by now; but they have not. The primordial fireball expanded and cooled too quickly to produce the heavier elements. Aeons of "stellar cooking time" were required to convert hydrogen and helium into heavier elements like oxygen, carbon, nitrogen, manganese, iron, and radon. The bodies of living things as we know them consist, in part, of elements synthesized only by nuclear reactions in the stars. The heavy elements were dispersed through the cosmos only when giant supernovas exploded. Their debris forms the planets of our solar system and others like them throughout the universe. This account of the origin and distribution of the heavy elements in and through the explosion of supernovas was dramatically confirmed in 1987 when, for the first time in the modern world, astronomers actually observed a supernova explosion in the relatively nearby Large Magellanic Cloud of stars. ${ }^{55}$ Late in 1992, astronomers detected another supernova explosion almost five billion light years away. The Hubble Space Tele- 
scope has since located many more in far away galaxies, and astronomers use them as "standard candles" for measuring vast cosmic distances.

Stellar cooking could not and did not produce the quantities of deuterium (heavy hydrogen) and helium that exist in the universe. Only the extreme temperatures, densities, and pressures that existed during the earliest phases of the Big Bang can account for them. Stars generate some helium at a rate that accounts for only 2 percent of all the helium in the universe. The Big Bang is required for all the rest. The Big Bang directly generated hydrogen, helium, and traces of deuterium and lithium. Irregularities in the primordial fireball eventually separated vast clouds of gas, which gravity then condensed into quasars and stars; and the stars later cooked up the heavy elements. Everything observable came out of the Big Bang, one way or another.

\section{F. The Cosmic Microwave Background}

Today's astronomers can "see" the universe in many ways. They view it through powerful optical telescopes; they listen to its radio waves; and they detect its radiant $\mathrm{X}$-rays, gamma rays, ultraviolet rays, and infrared rays. These types of radiant energy, coming to us from local and non-local regions of the universe, can now be converted to visual images. Radio waves furnish some of the most powerful evidence available for the Big Bang.

Prior to 1965 , many scientific cosmologists, especially those attracted to Steady State Cosmology (to be examined later) still had doubts about Big Bang Cosmology. A remarkable achievement of modern astronomy occurred in 1965, providing crucial evidence for the Big Bang. In 1965, two relatively new Ph.D.s, Arno Penzias and Robert Wilson, worked for Bell Laboratories in New Jersey on projects in radio astronomy. They tried to eliminate all extraneous sources of radio static from their instruments so they could obtain the purest possible signals from outer space, but they found that some noises could not be eradicated. These noises resembled the static we have all heard between stations on AM radio bands and the familiar snow between existing television channels; in fact about one percent of the photons that cause snow on TV belong to the microwave background that Penzias and Wilson discovered. To eliminate this radio hiss, they even shooed away pigeons and cleaned their droppings from their antenna. Nothing worked. No matter what they did, their radio telescope picked up rumbling noises from every direction. They did not realize at first that they had stumbled upon the still enduring echoes of the Big Bang.

Earlier cosmologists had predicted that a microwave background continued to exist as a remnant of the primeval explosion that created the universe. Some actively searched for it. As early as 1947, George Gamow and his associates predicted its existence at around 5 degrees Kelvin, which was off by two degrees, yet quite good for its day; but these predictions were ignored and forgotten. In the early 1960s, scientists in Russia and the United States again predicted 
its existence and calculated its temperature to be about 3 degrees Kelvin, the currently accepted figure. At Princeton University, astrophysicists under the leadership of Robert Dicke and P. J. E. Peebles were building a radio telescope to search for this microwave background; but Penzias and Wilson found it before the Princeton group completed construction. After Penzias and Wilson contacted the Princeton group, Dicke and Peebles visited their radioscope to listen. They realized that Penzias and Wilson had found what they were looking for, a cosmic radio hiss coming from everywhere in space. Most cosmologists now agree that the microwave background consists of continuing reverberations from the Big Bang. In 1978, Penzias and Wilson received a Nobel Prize for their discovery.

\section{G. The Dark Sky at Night}

The Big Bang theory of the origin of the universe is accepted for its explanatory power. It accounts for a vast amount of data that no other theory can explain-the receding galaxies and their redshift, Hubble's law of uniform expansion, the ongoing dissipation of energy and order within the world, Einstein's relativity theorems, the origin of the chemical elements, and the cosmic microwave background. It even accounts for something very commonplace that has puzzled astronomers since at least the middle of the eighteenth century: it explains why the sky is dark at night. Most of us have never wondered; the sun goes down; and that explains it. But if we live in an infinite universe, there is a puzzle, formulated first by the German physician Heinrich Olbers in 1826, and thereafter called "Olbers' Paradox."

Olbers knew perfectly well that the sun sets at night, but he wondered why there is not enough starlight to make the night sky as bright as day, or even as bright as the sun, assuming as Olbers did under the influence of Sir Isaac Newton, that the rest of the static but endless universe is as richly populated by stars as our visible universe. Imagine being at the center of a sphere surrounded by all the stars we can see. If we double the size of that visible sphere, as we might do with a more powerful telescope, stars will be only a fourth as bright, as predicted by Newton's inverse square law; but there will be four times as many of them to negate this effect. This doubling process could and should go on to infinity in an infinite universe until it encompasses an infinite number of stars. Light from each of these has had an infinite amount of time to reach us, so a star should shine in every last niche in the heavens, and the night sky should be as bright as the sun. Yet it is not. Why? Olbers thought that a gaseous medium filled the sky and blocked out most of the starlight; but this will not work. Given enough time, as Hermann Bondi showed, these gases would heat up and eventually radiate as much energy as they absorb..$^{56}$ And infinity is enough time!

Many attempts have been made to resolve Olbers' Paradox, but the most successful is provided by the Big Bang. Except for a few discussed in Chapter 
Three, today's cosmologists accept the Big Bang and agree that it provides the ultimate solution to Olbers' Paradox. Big Bang theory says that neither time nor space are infinite. Finite spacetime can contain only a finite number of stars that shine only for a finite amount of time. Stars come and go; they are born from cosmic debris, burn for a few billion years, exhaust their nuclear fuel, and die. The spatiotemporal finitude of the universe explains why most of the sky is dark at night and counts also as powerful evidence against the naturalistic claim that our universe is infinite in both space and time.

Additional evidence for the Big Bang origin of our universe will be presented in later chapters. To anticipate a bit, the hydrogen cycle, the gradual conversion of hydrogen into the heavier elements in supernova nucleosynthesis, could not have been going on forever, or there would be no hydrogen left in the universe; but there is. The same is true for all radioactive elements; if they have been losing electrons through nuclear decay for an infinite amount of time, no radioactive elements would remain today; but they do. The ripples in the microwave background discovered by the COBE satellite confirm the basic Big Bang scenario for galaxy and supergalaxy formation.

We have now reviewed the primary scientific evidence for Big Bang Cosmology; in Chapter Three we will consider putative evidence against it. If Big Bang Cosmology does not survive under critical scrutiny, there is no point in exploring our central philosophical question: What caused the Big Bang? Can natural science answer this question? Cosmological Agnosticism says that it cannot. Naturalistic and theistic metaphysicians give philosophical and religious answers to our question of ultimate causes, but there may be no scientific answer.

\section{Scientific Cosmological Agnosticism}

Scientists now advance a variety of explanations for the origin and evolution of the universe; but agnosticism says that we really do not know the answers. General Cosmological Agnosticism does not deny that many cosmological puzzles can be resolved. It focuses on the central question of this book: What caused the Big Bang?; and it says that we do not know. We know only what happened after the Big Bang was inaugurated, and science can not tell us what caused the Big Bang. Coming chapters will examine naturalistic theories that purport to offer scientific answers; but they actually give only highly speculative and dubious philosophical accounts of the ultimate origin of the universe, without acknowledging their subtle shift from science to unverifiable metaphysics. Scientific Agnostics claim that a plausible scientific account of the cause of the Big Bang is not and never will be available.

Agnosticism in cosmology may be either scientific or philosophical. Scientific Agnosticism says that the cause of the Big Bang lies beyond the limits of scientific methodology and knowledge. Philosophical Agnosticism extends 
this outlook. It says that the cause of the Big Bang lies entirely beyond the limits of philosophical knowledge, beyond all human reason, broadly understood. The remainder of this book will accept Scientific Cosmological Agnosticism, but total Philosophical Agnosticism will be rejected. Standard Big Bang Cosmology is inherently agnostic because it recognizes that scientific methodology and knowledge break down completely at or near the onset of the Big Bang. It makes no attempt to go behind the initial singularity or the earliest Planck dimensions.

If an initial singularity existed at $\mathrm{T}=0$, there were no laws of nature, no space, no time, and no physical causation. Cosmologists disagree about whether the breakdown of the laws of nature occurs at an initial singularity or at Planck dimensions; but most agree that they become inapplicable at some point. We can extrapolate scientifically all the way back to where these laws break down completely; but we cannot go beyond that because we must appeal to these laws just to get that far. Observable processes in nature like the redshift of the galaxies, the Hubble expansion, nucleosynthesis of the elements, the microwave background, and the dark night sky, all imply a Big Bang origin for the universe. Extrapolating from known laws of nature, these processes can be traced backward in time; but calculations result in zero time and space and infinite energy after eight to twenty billion years. Scientific Cosmological Agnostics insist that science itself cannot take us beyond and before that to a space beyond space, and a time before time began.

The fact that a world-creating Big Bang erupted billions of years ago is scientifically well established. The laws of nature can take us back to an initial singularity, but they can take us no further for at that point all physical laws break down completely and become inapplicable. The zero spacetime and infinite energy of the initial singularity indicate methodological failure as well as a cosmological beginning. Science cannot transcend its own limitations. With no laws of nature, spacetime, physical causation, or empirical data to work with, no further scientific extrapolations are possible. At an initial singularity, the ultimate limits of scientific knowledge are reached, the Scientific Cosmological Agnostic insists; and What caused the Big Bang? has no scientific answer. We cannot know what would make an initial singularity explode because it lies beyond the limits of all known laws of physics.

If a collapsing universe could shrink below Planck dimensions, the same agnosticism follows if all laws of nature become inapplicable at or below the Planck Wall. Later we will see that Quantum Cosmologists deny that anything can exist on the nether side of this Wall; if a sub-Planck collapsed universe existed, we could have no scientific knowledge of it because physical laws and causation appear for the first time at Planck dimensions. Many cosmologists are convinced that scientific knowledge terminates at the Planck Wall, that the Planck Wall is a blank wall. If the laws of nature only become applicable when the universe was $10^{-43}$ seconds old and $10^{-33}$ centimeters in diameter, then these 
dimensions represent the ultimate limits of scientific knowledge. What caused the appearance fifteen billion years ago of a universe with minimal Planck numbers? What caused the Big Bang? There are no scientific answers if, looking backward, scientific knowledge ends before these questions arise.

Theologians and philosophers may address the question of ultimate origins; but science has no answer, Scientific Cosmological Agnostics insists. Those scientists and naturalistic metaphysicians who affirm on allegedly scientific grounds that "Nature is eternal" must recant. Even "Every event has a natural cause" must be relinquished, for science ultimately reaches back to a point of origin beyond which no natural physical causes can be found. Natural causes are those that operate within our system of spacetime; but scientists generally agree that space, time, natural laws and physical causation began with the Big Bang and did not exist prior to either Plank dimensions or an initial singularity. Numerous prominent cosmologists and scientists today are Scientific Agnostics on the question of the cause of the Big Bang. ${ }^{57}$ In 1980, Robert Jastrow began his essay on "Science and the Creation," with these words:

I should like to say at the start that I am an agnostic in religious matters. I am fascinated, however, by some recent developments in astronomy-partly because of their religious implications and partly because of the peculiar reactions of my colleagues. In a nutshell, the astronomers, studying the universe through their telescopes, have been forced to the conclusion that the world had a beginning. Scientists have always felt more comfortable with the idea of a universe that has existed forever because their thinking is permeated with the idea of cause and effect; they believe that every event that takes place in the world can be explained in a rational way as the consequence of some previous event. If there is a religion in science, this statement can be regarded as its main article of faith. But the latest astronomical results indicate that at some point in the past the chain of cause and effect terminates abruptly. An important event occurred-the origin of the world-for which there is no known cause or explanation within the realm of science. ${ }^{58}$

Jastrow, convinced that science does not know what caused the Big Bang, insists that "We cannot find out what caused that beginning" because the scientist

comes to a blank wall where the big bang occurred. The cosmic explosion, the birth of the universe, is an effect for which he cannot find the cause. Some might say that if he cannot find it today, he will find it tomorrow; and we will read about it in the New York Times when Walter Sullivan gets around to it. This, however, is one finding in science that seems likely never to succumb to scientific investigation because in the first moments of the universe's existence the temperature and the pressure were infinitely 
high, which means that all relics of a pre-creation universe that might have given us a clue to some natural forces that conspired to bring about the explosive moment we call the big bang are gone. All of the evidence the scientist could examine to explain this cosmic holocaust has been melted down and destroyed in the fiery heat of that first moment itself. That is why it seems to me and a few other people that this is a blank wall, a curtain covering the mystery of creation never to be raised by human minds, at least in the foreseeable future.

This brings us to a very interesting pass. The world has come into being as a product of forces that are today, and very likely forever, outside the reach of scientific inquiry. These forces do not fit into the present body of natural forces-gravity, electricity, nuclear forces-and, being outside the realm of nature, as the scientists understand it, they must therefore, properly be termed supernatural. In this statement cosmologist and astronomer finally come face to face with the theologian, who has always thought that what one might call a supernatural force, a creative force, has been responsible for the origin of this world. ${ }^{59}$

If our system of nature is the only cosmos, the whole of physical reality, and if it was brought into being by causes that transcend nature, these causes must be classified as "supernatural." Were the supernatural causes of the Big Bang Divine? Not necessarily. In the next few chapters, we will consider metaphysical Naturalism and a variety of prestigious but pseudoscientific naturalistic cosmologists who contend that the transcendent causes of our universe were not Divine. Yet, if Scientific Cosmological Agnosticism is correct, their own metaphysics is no more verifiable or scientific than that of the theologians.

Standard Big Bang Cosmology affirms that scientific knowledge breaks down at the beginning of our cosmos, so there can be no scientific knowledge of what caused the Big Bang. The question must be turned over to philosophers and theologians. Can they do any better? In particular, can philosophical Naturalism or Theism do any better? We shall see in the following pages. In his 1978 book, God and the Astronomers, Robert Jastrow first takes us on a scientific journey through the evolution of the universe all the way back to the primordial cosmic fireball. Then, speaking from the vantage point of Standard Big Bang Cosmology, he identifies the final step that science takes in its cosmological inquiry into origins.

Now we would like to pursue that inquiry farther back in time, but the barrier to further progress seems insurmountable. It is not a matter of another year, another decade of work, another measurement, or another theory; at this moment it seems as though science will never be able to raise the curtain on the mystery of creation. For the scientist who has lived by his faith in the power of reason, the story ends like a bad dream. He has 
scaled the mountains of ignorance; he is about to conquer the highest peak; as he pulls himself over the final rock, he is greeted by a band of theologians who have been sitting there for centuries. ${ }^{60}$

Jastrow first wrote his book before the 1980s, when inflation and quantum physics were added to the Big Bang theory of origins. Chapters Four through Eight will consider more carefully whether Quantum Cosmologies can overcome the apparent limits of scientific knowledge by introducing quantum laws and effects at the beginning of our cosmic epoch.

In sum, Standard Big Bang Cosmology is agnostic on the question of ultimate cosmic or supercosmic causes. As a scientific theory, it deals with the evolution of the universe from either the initial singularity, or from Planck time, to today. It incorporates all empirical evidence that supports the theory; but it offers no scientific answer to: What caused the Big Bang? It turns this question over to agnostics, philosophers, and theologians. Can they do any better? We will see. Robert Jastrow suggests that this is "the most interesting question of all" about which to speculate. ${ }^{61}$ But it is speculation, not natural science. 